\title{
A theory for bone resorption based on the local rupture of osteocytes cells
}

\section{connections: A finite element study}

Ridha Hambli ${ }^{\mathrm{a},}{ }^{*}$, Khalid H. Almitani ${ }^{\mathrm{b}}$, Abdessalem Chamekh ${ }^{\mathrm{b}}$, Hechmi Toumi ${ }^{\mathrm{c}}$ and Joao Manuel R. S. Tavares $^{\mathrm{d}}$

${ }^{a}$ PRISME laboratory, EA4229, Polytechnic Institute of Orleans

Polytech' Orléans, 8, Rue Léonard de Vinci 45072 Orléans, France

ridha0007@yahoo.com

Corresponding author

${ }^{\mathrm{b}}$ King Abdulaziz University, Faculty of Engineering - Department of Mechanical Engineering, Saudia

${ }^{c}$ University of Orleans, I3MTO Laboratory, EA 4708, Hospital of Orleans, 1 rue Porte Madeleine, F-45032, Orléans, France

d Instituto de Engenharia Mecânica e Gestão Industrial, Departamento de Engenharia Mecânica, Faculdade de Engenharia, Universidade do Porto, Rua Dr. Roberto Frias s/n, Porto, Portugal

\begin{abstract}
In this work, a bone damage resorption finite element model based on the disruption of the inhibitory signal transmitted between osteocytes cells in bone due to damage accumulation is developed and discussed. A strain-based stimulus function coupled to a damage-dependent spatial function is proposed to represent the connection between two osteocytes embedded in the bone tissue. The signal is transmitted to the bone surface to activate bone resorption. The proposed model is based on the idea that the osteocyte signal reduction is not related to the reduction of the stimulus sensed locally by osteocytes due to damage, but to the difficulties for the signal in travelling along a disrupted area due to microcracks that can destroy connections of the intercellular network between osteocytes and bone-lining cells. To check the potential of the proposed model to predict the damage resorption process, two bone resorption mechano-regulation rules corresponding to two mechanotransduction approaches have been implemented and tested: 1) Bone resorption based on a coupled strain-damage stimulus function without ruptured osteocyte connections (NROC); and 2) Bone resorption based on a strain stimulus function with ruptured osteocyte connections (ROC). The comparison between the results obtained by both models, shows that the proposed model based on ruptured osteocytes connections predicts realistic results in conformity with previously published findings concerning the fatigue damage repair in bone.
\end{abstract}

Keywords: Bone, Fatigue, Cracks, Ruptured osteocytes, Finite element model, Remodeling 


\section{Introduction}

The adaptation of bone to cyclic loads involves a complex physiological response that is targeted to local zones of damage. It has been suggested that osteocytes produce a signal proportional to mechanical loading by sensing strain and fatigue microcracks and transmit signals activating remodeling to remove bone where the damage is excessive [1-3]. It is widely accepted that the mechanotransduction of stimuli in bone by osteocytes is governed by several possible events, including shear stress along dendritic processes and/or the cell body, cell deformation in response to strain, and primary cilia $[4,5]$. These events could occur independently or simultaneously to activate mechanotransduction.

Osteocytes are capable of transducing changes in mechanical stimuli into biochemical signals that regulate cellular responses $[6,7]$. Frost [8] originally proposed that remodeling would occur to repair damage in bone. He suggested that the disruption of canalicular connections that occurred when cracks crossed them could provide the stimulus to initiate remodeling. Disruption of the inhibitory signal transmitted due to damage was proposed by Martin et al. [9] and has been considered by several authors as a fundamental mechanism that governs bone remodeling $[4,10$ 12]. Several finite element models have been developed to simulate the bone remodeling process, including bone resorption due to damage using local strain-damage stimuli functions [3, 13-15]. A limitation of these studies is that the osteocyte network inhibitory signal transmission due to damage accumulation within the bone tissue has not been incorporated into finite element models including opening and closure of cracks. It is agreed that osteocyte networks are the best candidates to transduce changes in strain and microdamage. They are thought to transmit a biochemical, electrical or any other type of signal to the lining cells at the bone surface [16].

Garcia-Aznar et al. [15] proposed a finite element remodeling model based on a bone local stimulus function expressed in terms of a signal generated by the strain and the local bone damage sensed by osteocytes. The authors suggested that any local perturbation in the reception of this signal causes a certain degree of osteoblast/osteoclast activation, and they concluded that it is important to know how this signal is induced by strain and microdamage. 
Several 2D simplistic studies considering the osteocytes network were proposed to develop models for real-time signaling induced in osteocytic networks by mechanical stimuli [17] and to simulate the signal loss due to osteocyte apoptosis [18].

In this work, a bone resorption finite element model based on the concept of inhibitory signal transmission rather than on a local stimulus function has been developed and implemented in ABAQUS (Dassault Systèmes, USA). The model is based on the following two novelties: 1) At each bone site, osteocytes transduce locally the strain stimulus; and 2) A particular shape of a damage-dependent spatial function representing the connection between an osteocyte and the bone surface 'weighted' by the damage value inhibits signal transmission to the bone lining cells in the surface which can trigger bone resorption. The finite element model is based on the idea that the osteocyte signal reduction is not related to the reduction of the stimulus sensed locally by osteocytes due to damage, but to the difficulties for the signal in travelling along a disrupted area. To check the potential of the proposed model to predict the bone resorption due to damage accumulation, two mechano-regulation laws have been implemented and tested, corresponding to two mechanotransduction approaches: (i) Bone resorption based on a coupled strain-damage stimulus function without ruptured osteocyte connections (NROC), as proposed by García-Aznar et al. [15]; and (ii) Bone resorption based on a strain stimulus function with ruptured osteocyte connections (ROC).

The fundamental difference between the proposed model and previously published ones $[3,11,13-$ $15,19]$ is that the damage affects the signal transmission sensed by the osteocyte network expressed in terms of a damage-dependent spatial function rather than a local coupled strain-damage stimulus function. Also, contrary to previous works, the new model considers the difference between the damage accumulation effects in tension and compression in signal transmission to trigger bone resorption.

A further feature of the proposed model is that it will make it possible in future work to link the signal distribution to the biophysical mechanisms governed by the fluid transport within the pericellular matrix space of the lacunar-canalicular system. 


\section{Theory for fatigue damage modelling of bone}

Taylor et al. [12], using a combined continuum damage mechanics and finite element approach, proposed a preliminary study to simulate the fatigue behaviour of cancellous bone based on the assumption that the fatigue behaviour of trabecular bone is similar to that of cortical bone.

When dealing with loading histories composed of well-defined discrete cycles, an evolution law in terms of the number of cycles and their amplitudes is often considered more practical in the literature. The number of cycles $N$, is then regarded as a continuous time-like variable and the growth of damage, which occurs during discrete time intervals within a cycle, is spread over the entire cycle in a process of continuous evolution. Such a cycle-based formulation can be obtained in the form of:

$$
\frac{\partial D}{\partial N}=f\left(\frac{N}{N_{f}}\right),
$$

where $N_{f}$ denotes the cycle at failure.

Integrating Eq. (1), leads to Chaboche's model [20] expressed by:

$$
D=1-\left[1-\left(\frac{N}{N_{f}}\right)^{\frac{1}{1-\alpha}}\right]^{\frac{1}{1+\beta}} .
$$

The cycle at failure $N_{f}$ can be computed using the experimental results presented in [11] that distinguish between compression and tension loads, respectively:

$N_{f}^{c}=1.479 \times 10^{-21} \Delta \varepsilon^{-10.3}$ for compressive loads,

$N_{f}^{t}=3.630 \times 10^{-32} \Delta \varepsilon^{-14.1}$ for tensile loads,

where $\Delta \varepsilon$ is the range of the applied microstrain.

The theory of continuum damage mechanics $[14,20]$ suggested that the damage variable can be considered as an index ranging from $O$ to $D_{R}$ quantifying the local damage state of the material (Fig.1). 
$\begin{cases}D=0 & \text { Bone tissue is free from damage } \\ 0<D<D_{R} & \text { Bone tissue is damaged } \\ D=D_{R} & \text { Bone tissue is completly cracked }\end{cases}$

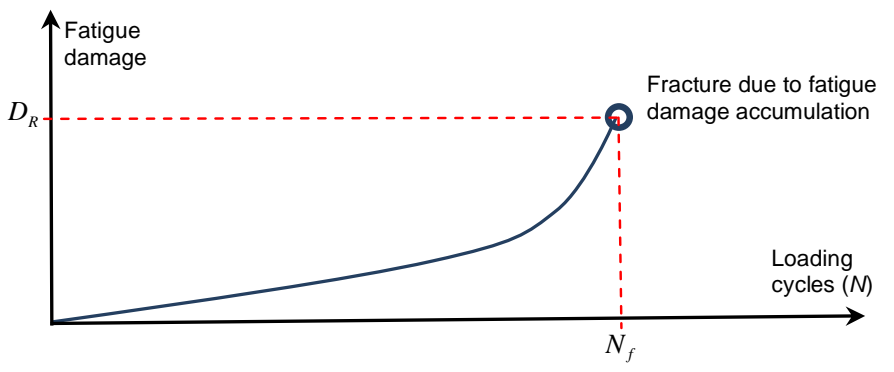

Figure 1. Illustration of fatigue damage growth. $D_{R}$ is the critical damage value at which fracture occurs and $N_{f}$ is the cycle at failure.

\section{Bone-damage resorption model}

Two bone resorption mechano-regulation rules corresponding to two mechanotransduction approaches have been implemented and tested: (i) Bone resorption based on a coupled straindamage stimulus function without ruptured osteocyte connections (NROC); and (ii) Bone resorption based on a strain stimulus function with ruptured osteocyte connections (ROC). Figure 2 illustrates the fundaments of NROC and ROC models.
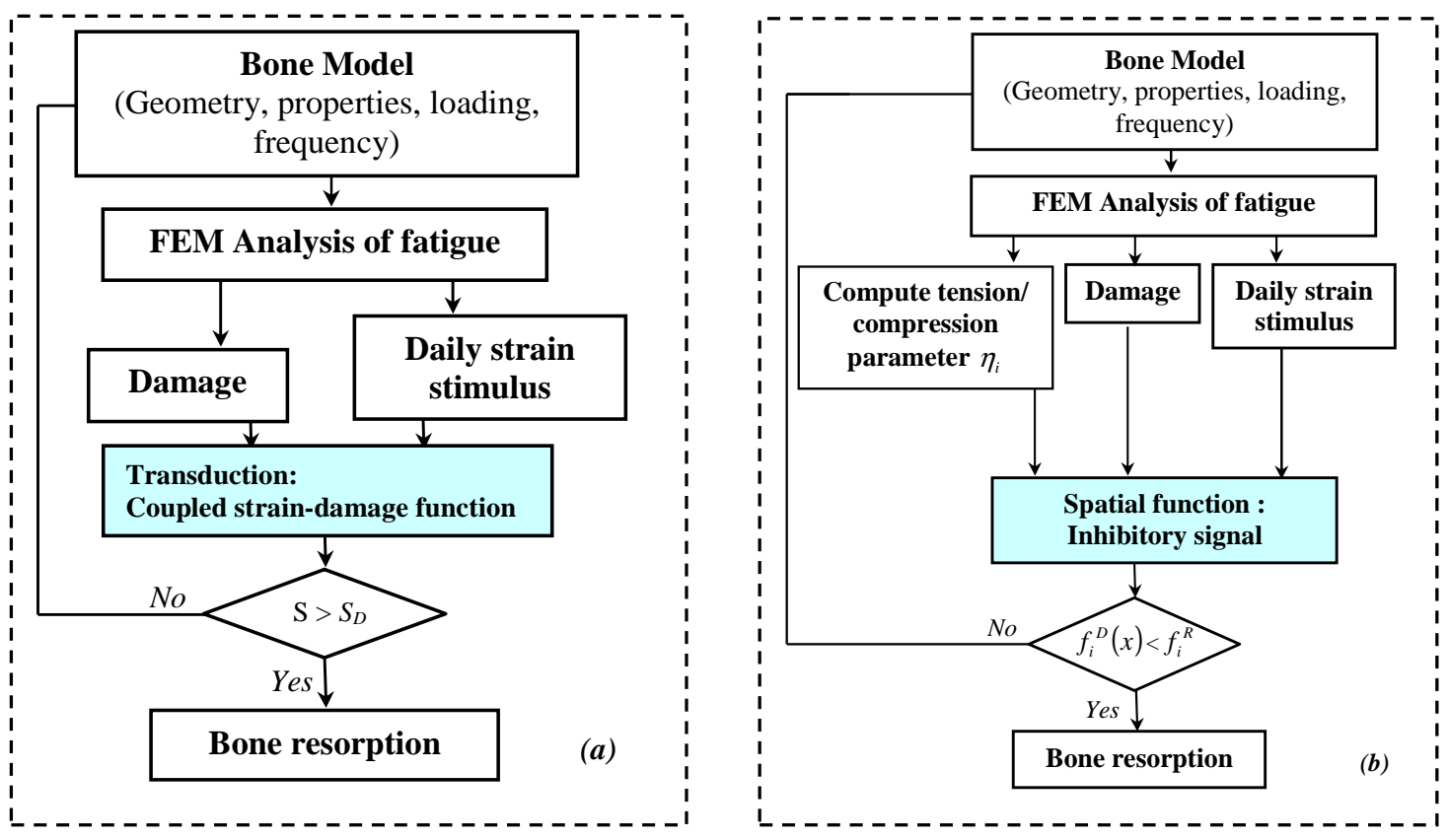

Figure 2. Fundaments of conventional local coupled strain-damage with (a) Non ruptured osteocytes processes signal (NROC) model and (b) proposed ruptured osteocytes Signal (ROC). 
In the case of model NROC: the resorption is triggered when the damage exceed locally a critical value (Equ. 8). In the case of model ROC: the resorption is triggered when the osteocytes connections are broken because damage (Equ. 20)

\subsection{Model based on non-ruptured osteocyte processes (NROC)}

Bone is a multiscale composite material composed of a tissue matrix and pores (voids). Remodelling theories consist in the description of the time evolution of the different phases (bone and pores). Several approaches to remodelling activated by microdamage have been proposed [3, $11,14-15,19]$. Most of these models are phenomenological and describe the resorption process based on local damage theories. The models suggest that resorption occurs when a strain-damage stimulus function reaches its critical value corresponding to a pathological strain of $3500 \mu \varepsilon$ [3]. Resorption is then triggered locally or in the bone surface without taking into account the signal alteration through the cellular network due to the existence of microcracks that disrupt the network connectivity.

In continuous models for trabecular bone modeling, reduction/apposition of the bone mass is associated to an increase or a reduction in porosity. In fact, remodelling in cancellous bone is known to take place always on the surfaces of macrocopic voids. The application of a spatial function ensures that the remodeling process occurs at the bone surface and hence affects the porosity of the trabecular bone.

In the present work, a phenomenological averaged isotropic continuum model has been used to describe the resorption process based originally on McNamara and Prendergast [3] extended by Hambli et al. $[14,19]$. The primary focus here was to implement a model based on ROC to the bone surface to trigger resorption and check its validity.

The model described by Equations (5-8) is represented schematically in Figure 3. 


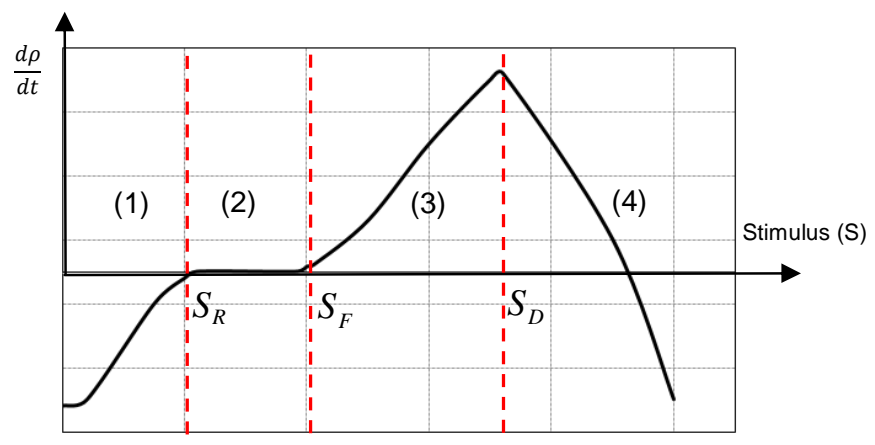

Figure 3. Bone adaptation model based 4 phases: (1) Resorption due to disuse, (2) Lazy zone where bone mass is kept constant, (3) Formation due to strain stimulus dominated, (4) Resorption due to fatigue damage (Bone repair) (adapted from [3, 14, 19].

The set of equations in relation of the model of Fig. 3 describing the change in bone density is given in [19] as:

\begin{tabular}{|l|l|}
\hline \multicolumn{1}{|c|}{ Remodeling state } & \multicolumn{1}{|c|}{ Equation } \\
\hline Resoption due to disuse & $\frac{d \rho}{d t}=\alpha_{R}\left(S-S_{R}\right), \quad$ If $\quad S<S_{R}$ \\
\hline Lazy zone (no formation and no resorption) & $\frac{d \rho}{d t}=0, \quad$ If $\quad S_{R} \leq S \leq S_{F}$ \\
\hline Formation due to over use & $\frac{d \rho}{d t}=\alpha_{F}\left(S-S_{F}\right), \quad$ If $\quad S_{F}<S<S_{D}$ \\
\hline Resoption due to damage repair & $\frac{d \rho}{d t}=\alpha_{D}\left(S-S_{D}\right)$, If $\quad S>S_{D}$, for the NROC model \\
\hline
\end{tabular}

where $\rho$ is the bone relative density, $t$ is time, $\alpha_{R}, \alpha_{F}$ and $\alpha_{D}$ denote the bone resorption rate due to disuse, forming rate and the bone resorption rate due to damage accumulation.

$S, S_{R}, S_{F}$ and $S_{D}$ denote remodeling stimulus, set point of bone resorption due to disuse, set point of bone formation and set point of damage resorption, respectively.

It's important to note that Equ.(5) describes resorption due to disuse resulting from low strain level applied to bone site by inhibition of the stimulatory signal, while Equ. (8) describes resorption due to excessive damage (damage repair).

The new density value of the bone tissue is approximated using the forward Euler method by:

$$
\rho_{t+\Delta t}^{i}=\rho_{t}^{i}+\Delta \rho^{i} .
$$


Combining damage definition and elastic modulus evolution in the framework of bone remodeling, the elastic modulus $E$ at each bone location is calculated from the density, the bone mineralization and the damage according to Garcia-Aznar et al. [15]:

$$
E=C(1-D) \rho^{p} \alpha^{q}
$$

where $C, p$ and $q$ are experimentally derived constants, and $\alpha$ is the ash function that denotes the degree of bone mineralization expressed by Hernandez [21]:

$$
\alpha(t)=\alpha_{\max }+\left(\alpha_{0}-\alpha_{\max }\right) e^{-k t}
$$

with $\alpha_{0}, \alpha_{\max }$ and $k$ denoting initial mineralization, maximum degree of mineralization and a parameter determining the shape of the temporal evolution curve, respectively.

Mullender and Huiskes [22] assumed that the mechanical stimulus is sensed by sensor cells (osteocytes) which initiate bone remodeling. From the finite element point of view, each element of the mesh contains a 'sensor' cell placed in its centroid. The effect of the centroid signal on bone remodeling decreases as the distance between the sensor cells and the bone surface location increases. The relative density at each location $i$ is regulated by the local stimulus value $S_{i}$ to which all osteocytes contribute relative to their distance expressed by:

$S(x, t)=\sum_{i=1}^{N_{o c}} f_{i}(x) \mu_{i} S_{i}$ for the non-damage spatial function model (NROC),

where $\mu_{i}$ is the mechanosensitivity of the osteocyte $i, N_{o c}$ is the number of osteocytes, and $f_{i}(x)$ is the non-damage spatial function.

Several stimuli functions have been proposed by various authors to describe the transduction process. In this work, a daily strain history based stimulus was used for the NROC model as suggested by Garcia-Aznar et al. [15]:

$$
S_{i}=\frac{\xi}{\xi+c}(1-D)^{a}
$$


where $a$ and $c$ are parameters that define the transduction role of damage and strain variables, and $\xi$ is the daily strain history proposed in [23]:

$$
\xi=\left(\sum_{j} N_{j} \varepsilon_{j}^{m}\right)^{\frac{1}{m}}
$$

with $N_{j}$ denoting the number of load cycles per day, $\varepsilon_{j}^{m}$ the level of the applied strain for each load case $j$, and $m$ the stress exponent that weights the relative importance of the strain magnitude and the number of load cycles in the mechanical stimulus.

The spatial function $f_{i}(x)$ for the NROC model was defined by Mullender and Huiskes [22] as:

$$
f_{i}(x)=\exp \left(-d_{i}(x) / d_{0}\right)
$$

where $d_{i}(x)$ is the distance between the osteocyte $i$ and the bone surface location $x$, and the parameter $d_{0}$ represents a normalization factor that limits the area of influence of the osteocyte.

Figure 4 shows the plot of the spatial function $f(x) \quad$ (Equ. 15) which depends only on the distance between the osteocyte $i$ and the bone surface location $x$. In can be noticed that that damage level do not affect the amplitude of the function.

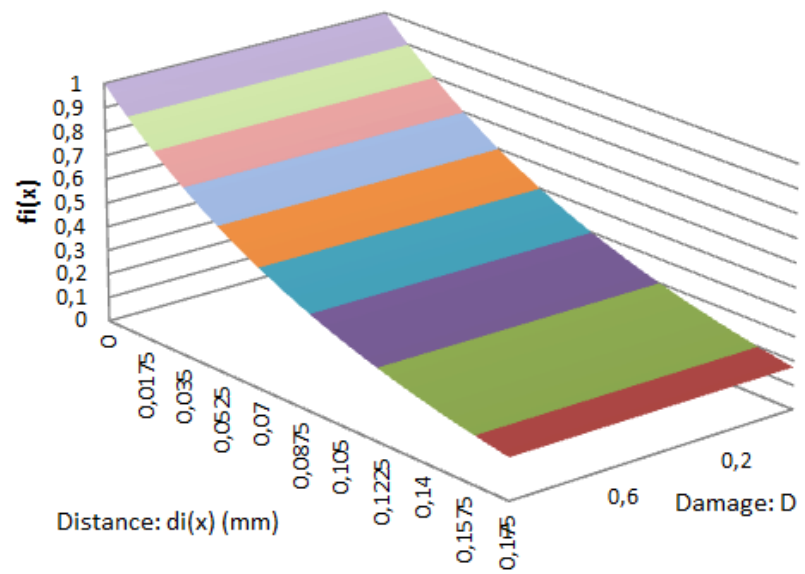

Figure 4. Plot representing the function $f(x)$ (Equ.15) in the case non-ruptured osteocyte processes (NROC). 


\subsection{Model based on ruptured osteocyte processes (ROC)}

In the case of ROC model, we propose a stimulus function expressed by:

$S(x, t)=\sum_{i=1}^{N_{o c}} f_{i}^{D}(x) \mu_{i} S_{i}$

$f_{i}^{D}(x)$ is the proposed damage-dependent spatial function (ROC model).

The development of the ROC model is based on the following two concepts:

1) Osteocytes transduce the strain stimulus locally at each bone site.

2) A particular shape of the damage-dependent spatial function representing the connection between an osteocyte and the bone surface 'weighted' by the damage value inhibits signal transmission to the bone lining cells in the surface that can trigger bone resorption.

Depending on the position of an octeocyte, Mullender's model suggests that the only factor which affects bone surface remodeling is the distance $d_{i}(x)$. Furthermore, the degree of damage seems to directly influence the initiation of bone remodeling. Martin [11] suggested that a major part or even all bone remodeling is induced by damage. Certainly, microcracks can destroy connections of the intercellular network between osteocytes and bone-lining cells. This and other items give rise to the ROC model. As outlined by Martin [6], a permanent stress-generated signal is emitted by these cells in order to inhibit the activity of osteoclasts. A microcrack would thus interrupt the transmission of such a signal, causing the activation of remodeling (Fig. 5). 


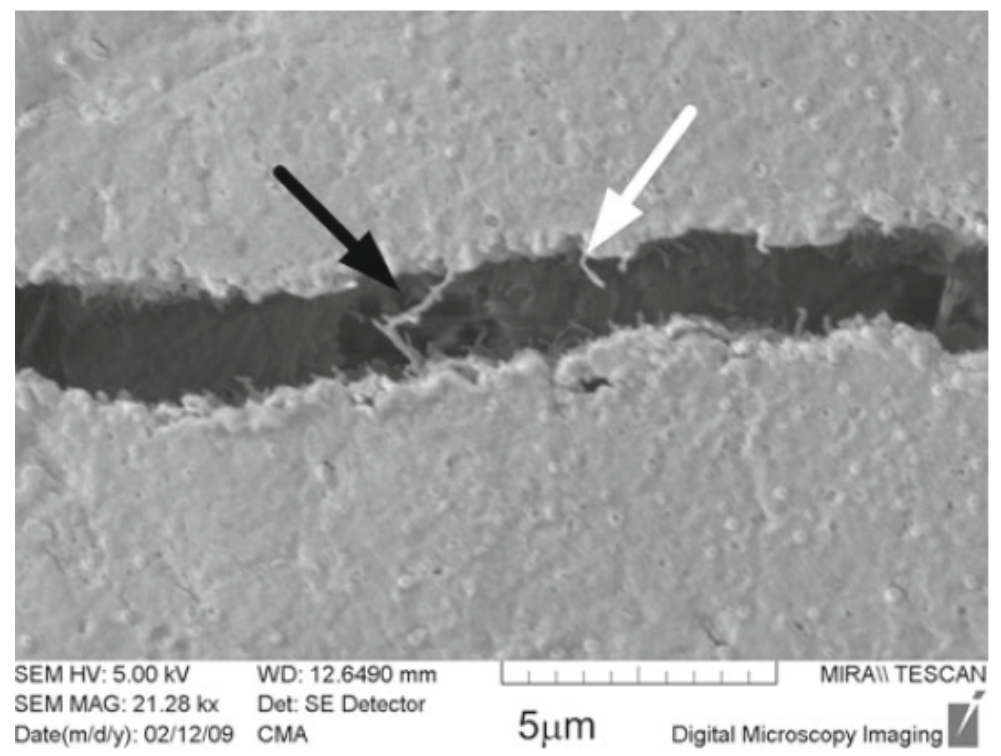

(a)

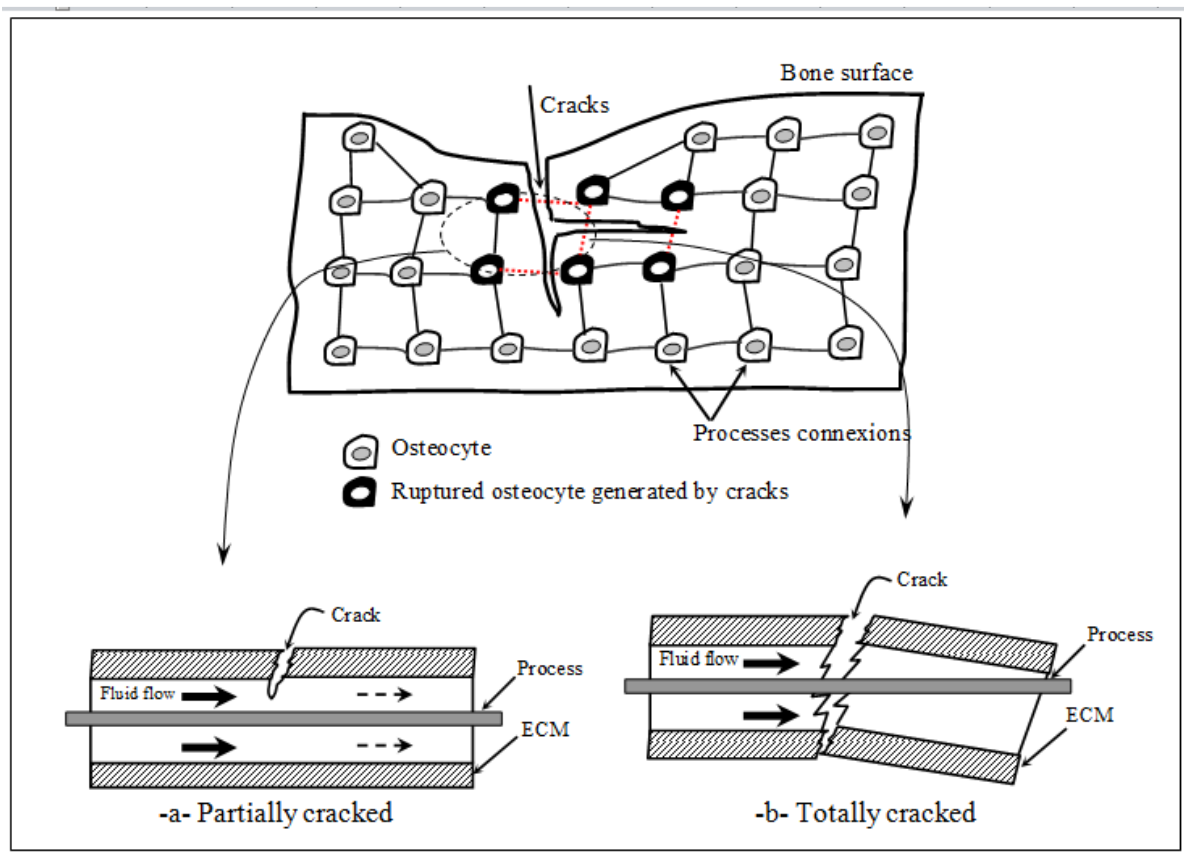

(b)

Figure 5. (a) Scanning electron microscope image showing part of a crack, with an intact process (black arrow) and an incomplete process (white arrow) passing across between the crack faces (adapted from [24]). (b) Schematic diagram of bone to illustrate the inhibitory signal transmitted by the osteocyte due to crack accumulation under excessive load (ECM: Extracellular matrix). The multiple thin lines connecting the osteocytes represent cell processes within canaliculi. The cracks generate the rupture of osteocyte connexions, inhibiting the osteocytic signal transmission to osteoblasts and activating the osteoclasts to remove the cracked area.

Based on the previous observations, disruption of the transmitted signal due to damage in the osteocyte network can be modelled by a reduction parameter depending on the average damage 
between the osteocyte and the bone surface (resorption sites). Hence, instead of a non-damage distance function $d_{i}(x)$, one can consider a damage distance function $d_{i}^{D}(x)$ in the Mullender spatial function. Therefore, we can adopt here a spatial function $f_{i}^{D}(x)$ 'weighted' by the damage distance function $d_{i}^{\text {Dam }}(x)$ value between an osteocyte and the (surface) in the form:

$$
f_{i}^{D}(x)=\exp \left(-d_{i}^{\text {Dam }}(x) / d_{o}\right)
$$

where $d_{i}^{\text {Dam }}(x)$ can be interpreted as the damage-dependent distance between the osteocyte $i$ and the bone surface location $x$. Also, it is much more physics-based approach to modify the exponential parameter $d_{i}(x)$ in Eq. 9 by including $d_{i}^{\text {Dam }}(x)$ distance and a crack closure/opening damage parameter, in order to consider the difference between damage accumulation effects in tension and compression during bone resorption.

Here, we propose the following expression of $d_{i}^{\text {Dam }}(x)$ distance:

$$
d_{i}^{\text {Dam }}(x)=\frac{d_{i}(x)}{\left(1-\eta_{i}\right)^{K}}
$$

where $\kappa$ is a damage exponent that expresses the mechanosensitivity of the osteocyte process $i$ in transmitting the damaged signal, and $\eta_{i}$ is a crack closure/opening damage parameter. The concept of continuous crack closure/opening consists in the expression of parameter $\eta_{i}$ suggested in [25]:

$$
\eta_{i}=\left(\eta_{c}+\left(1-\eta_{c}\right) \frac{\sum \sigma_{p}^{+}}{\sum\left|\sigma_{p}\right|}\right) D_{i},
$$

where $D_{i}$ is the damage at location $i$ given by Eq. (2), $\eta_{c}$ stands for the tensile/compression parameter with $0<\eta_{c} \leq 1$, and $\sigma_{p}^{+}$and $\sum\left|\sigma_{p}\right|$ denote the positive and the sum of the absolute values of the principal stresses.

Figure 6 shows the plot of the spatial function $f_{i}^{D}(x)$ (Equ. 17) which depends on both distance between the osteocyte $i$ and the bone surface location $x(d(x))$ and a representation of the 
damage state by a continuous crack closure/opening parameter $\eta_{i}$ (Equ. 19). In can be noticed in this case that the damage level have a strong effect on the amplitude of the damaged spatial function $f_{i}^{D}(x)$ compared to the non-damaged one $f(x)$ (Fig. 4).

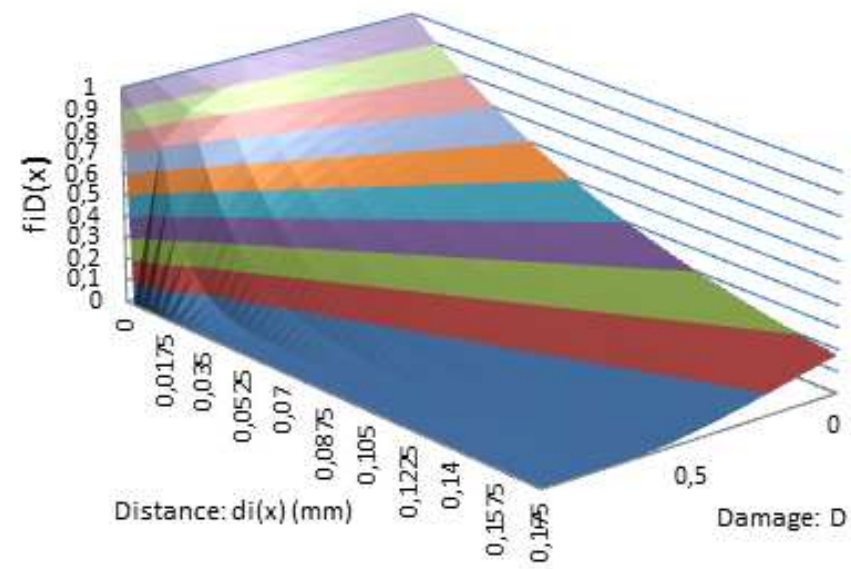

Figure 6. Plot representing the function $f_{i}^{D}(x)$ (Equ.17 and 18) in the case ruptured osteocyte processes (ROC).

In the case of ROC model, the level of the transmitted signal expressed by the distance function $f_{i}^{D}(x)$ depends on the damage value modulated by the term $d_{i}^{\text {Dam }}(x)$ (Equ. 18) contrary to the case of NROC model where the signal function $f_{i}(x)$ is independent from damage.

It has been hypothesized that bone remodeling is controlled at the cellular level through signals mediated by osteocytes [25]. This hypothesis has been supported by results from in vitro experiments by You et al. $[26,27]$ in which bone cells were subjected to a fluid flow generated by shear stresses. The results of an in vivo experimental study by Vashishth et al. [28] support the sensory role of osteocytes in human cortical bone and the decline of the osteocyte lacunar density caused by an accumulation of microcracks. Therefore, the resorption condition can be expressed by: $f_{i}^{D}(x) \leq f_{i}^{R}$ 
where $f_{i}^{R}$ denotes the reference value of the transmitted signal triggering the osteoclast activity for bone resorption. This value can be related to the threshold strain amplitude (3500 $\mu \mathrm{m})$ for damage accumulation in bone as reported in $[3,19]$.

Note that, (i) in the case of the NROC model, Eq. (8) describes the rate of bone resorption due to local damage accumulation when the damage exceeds its critical value $D_{R}$ of about $2.6 \mathrm{E}-10$ [3, 19] (Fig. 2) and (ii) in the case of the ROC model, Eq. (8) is substituted Eq. (20) in the constitutive equations to represent the resorption due to excessive damage by the rupture of osteocyte processes.

\subsection{Surface resorption condition algorithm}

During remodeling, osteoclasts travel along the surface of the bone resorbing tissue and forming a resorption cavity [16]. Therefore, in the current model the resorption was restricted to bone surface in order to remove the damage. In the current work, a surface remodeling condition was implemented to ensure that bone resorption occurs at the bone surface. This implies that bone can be removed only from surface of the trabeculae during the crack repairing process. i.e. the stimulus coupled to damage is calculated at the bone volume and adaptation occurs accordingly at the bone surface located at the adjacent the damaged zone.

The bone surface condition is described by the following steps (Fig. 7):

- at every FE $(i)$ of the mesh, the algorithm compute the number $\left(n_{\text {surr }}\right)$ of the FE surrounding of the FE (i),

- $\quad n_{\text {surr }}<4$ : the element is located at the bone surface and may be resorbed

- $n_{\text {surr }}=4$ : the element is located in the bone volume and may not be resorbed 


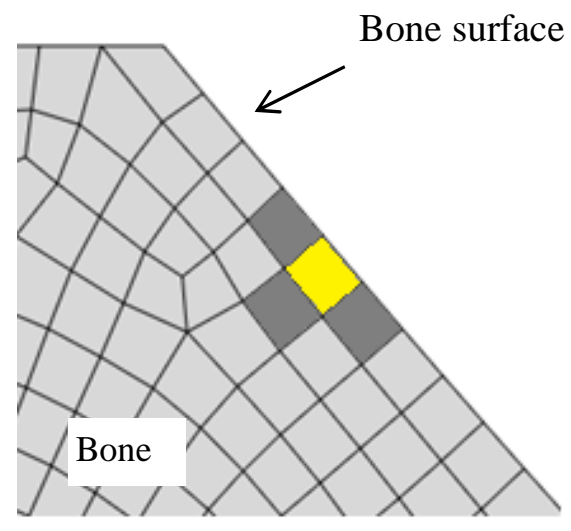

(a) Surface element (in yellow) which may. be resorbed: $n_{\text {surr }}<4$.

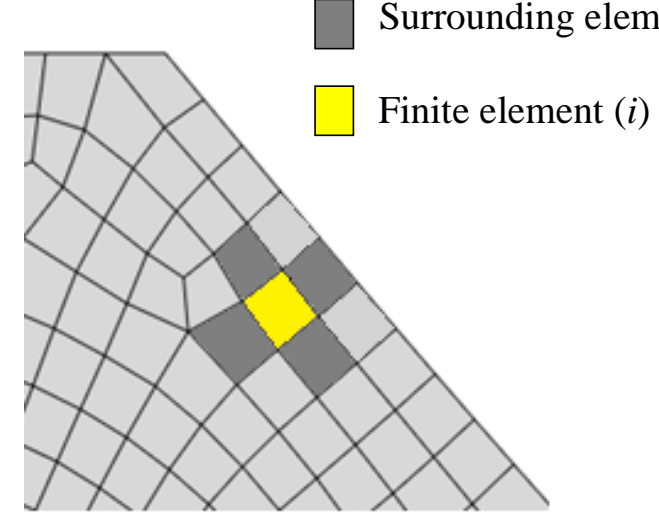

(b) Volume element (in yellow) which be may not be resorbed $n_{\text {surr }}=4$.

Figure 7. The surface remodeling condition procedure: at every FE (i) of the mesh, the algorithm compute the number $\left(n_{\text {surr }}\right)$ of the FE surrounding of the FE $(i)$.

The resorption surface condition is implemented for both models (ROC and NROC) to ensure that resorption starts at bone surface to reach the damaged area located deep in the bone.

\section{Simulation of bone resorption}

Macroscopic trabecular bone is a complex three-dimensional structure formed by single trabeculae. As a first approximation and for simplicity, the geometry of a trabecula can be defined as a circularshaped volume composed of a number of periodic unit cells each containing a cavity representing an osteocyte lacuna (Figure 8a). The circular section made by the cut A-A in Figure 8a is assumed to be the rectangular section shown in Figure $8 \mathrm{~b}$. The aim is not to simulate the response of real trabeculae with existing microcraks. The focus was to check whether the proposed NROC model is able to predict resorption bone patterns coherent with the concept of bone damage repair triggered by accumulation of fatigue damage.

Hazenberg et al. [2] have shown that microcracks in bone cause damage to the cellular material, by rupturing cell processes due to crack opening and shear displacements. Hence, in order to compare the proposed (ROC) model to the conventional (NROC) model, the remodelling of a pre-cracked trabecular bone area of $125 \times 100 \mu m$ (Figure 8c) was simulated using a 2D finite element analysis 
under shearing loading. A pre-crack was introduced in order to control the crack path during the loading and provoke a rupture by shearing (Figure 8c).

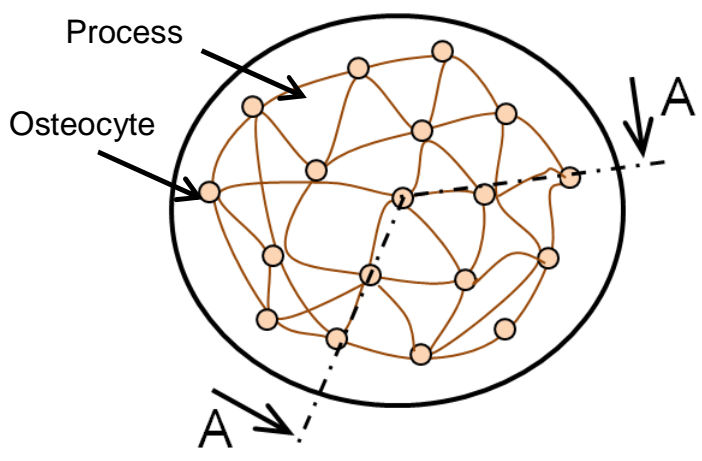

(a)

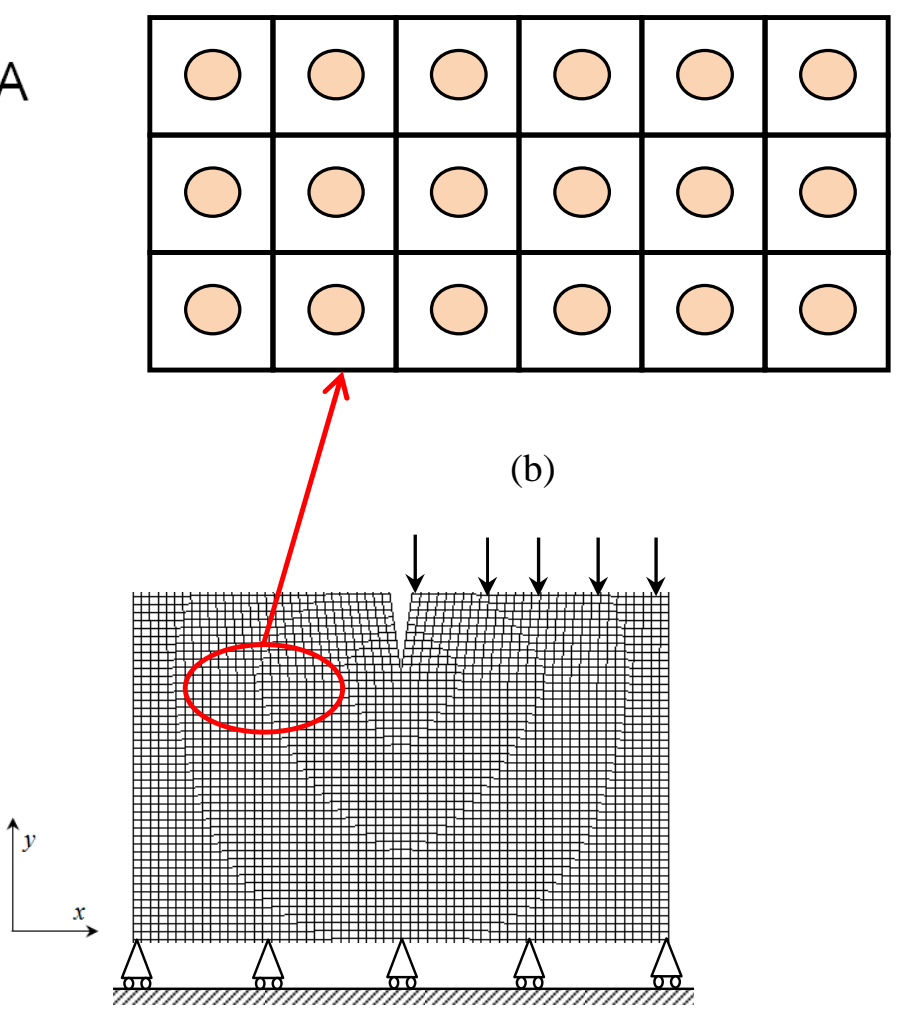

(c)

Figure 8. (a) Cross section representing the structure of a trabecula. (b) The unit of the periodic distribution assumption. (c) 2D Finite element mesh of unit cell model containing a notch (precrack) on the bone surface to provoke controlled rupture.

A mesh is generated using four-node plane strain elements. Each element of the mesh $i$, contains 'sensor' placed into its centroid representing an osteocytes density. Using one osteocyte centred at each finite element makes the problem mesh dependent. From finite element point of view and to avoid mesh dependent problems, we define in the present work an (osteocyte density)/(FE volume or area) ratio on every finite element of the mesh. In this case, the local stimulus computed on every finite element is weighted by its size.

The weighted local stimulus can be expressed by:

$$
\bar{S}_{i}=\frac{\bar{N}_{o c} S_{i}}{A_{i}^{F E}}
$$


$S_{i}$ and $A r_{i} E$ respectively the local stimulus computed at every integration point and the finite element volume (or area) at location $i$.

$\bar{N}_{o c}$ denotes the osteocytes density by volume expressed by :

$$
\bar{N}_{o c y}=\left\{\begin{array}{l}
N_{o c} / N_{r e f} / \mathrm{mm}^{3} \text { in case of } 3 D \text { problem } \\
N_{o c} / N_{r e f} / \mathrm{mm}^{2} \text { in case of } 2 D \text { problem }
\end{array}\right.
$$

$N_{o c}$ is the number of osteocytes/volume (or area for 2D problems) of bone which depends on the age and $N_{\text {ref }}$ is the reference number of osteocyte density for adults. [29] reported that in case of 3D measurements:

$$
10500 \leq N_{\text {ref }}^{3 D} \leq 13300 \text { osteocytes } / \mathrm{mm}^{3}
$$

In case of $2 \mathrm{D}$ problem, one can estimates that $\left(N_{r e f}^{\mathcal{D}}=N()_{r e f}^{3 D} \stackrel{2}{=}\right)$ :

$$
\begin{aligned}
& 479 \leq N_{\text {ref }}^{2 D} \leq 561 \text { osteocytes } / \mathrm{mm}^{2} \\
& N_{\text {ref }} \text { is expressed by: } \\
& N_{\text {ref }}=\begin{array}{l}
N_{j \text { ref }}^{3 D} \text { in case of } 3 D \text { problem } \\
N_{r e f}^{2 D} \text { in case of } 2 D \text { problem }
\end{array}
\end{aligned}
$$

$\rightarrow$ direction applied to the bottom edge as illustrated in Figure 8. An axial cyclic load of $10 \mathrm{~N}$ of 5000 cycles/day during 120 days was applied. This value generates in the finite element model a pathological strain of $3700 \mu \mathrm{m}$ which is above the $3500 \mu \mathrm{m}$ reported by McNamara and Prendergast [3] as the threshold strain amplitude for damage accumulation in bone. The bone material parameters used are indicated in Table 1. 


\begin{tabular}{|c|c|c|c|}
\hline Parameters & $\begin{array}{l}\text { Notation } \\
\text { (units) }\end{array}$ & Value & References \\
\hline \multicolumn{4}{|l|}{ General parameters } \\
\hline Initial Elastic modulus & $E_{0}(\mathrm{MPa})$ & 17000 & Proposed \\
\hline Poisson ratio & $v$ & 0.3 & {$[14,19]$} \\
\hline Initial density & $\rho\left(\mathrm{g} / \mathrm{cm}^{3}\right)$ & 1.5 & Proposed \\
\hline Density coefficient & $C\left(\mathrm{~g} / \mathrm{cm}^{3}\right)$ & 4000 & [15] \\
\hline Density exponent & $\gamma$ & 3 & [15] \\
\hline Compression parameter & $\eta_{c}$ & 0.5 & [25] \\
\hline Stress exponent & $m$ & 4 & [15] \\
\hline Stimulus parameter for NROC model & $a$ & 10 & Proposed \\
\hline Stimulus parameter for NROC model & $C$ & 0.0025 & Proposed \\
\hline \multicolumn{4}{|l|}{ Mineralization parameters } \\
\hline Mineralization law exponent & $Q$ & 2.74 & \multirow{6}{*}[15]{} \\
\hline Density law exponent & $P$ & 2.6 & \\
\hline Initial mineralization & $\alpha_{0}$ & 0.6 & \\
\hline Minimum degree of mineralization & $\alpha_{\min }$ & 0.4 & \\
\hline Maximum degree of mineralization & $\alpha_{\max }$ & 0.7 & \\
\hline Parameter of mineralization evolution & $k\left(\right.$ day $\left.^{-1}\right)$ & 0.000339 & \\
\hline \multicolumn{4}{|l|}{ Damage law parameters } \\
\hline Fatigue parameter & $\alpha$ & 0.2 & \multirow{3}{*}[14,19]{} \\
\hline Fatigue exponent & $\beta$ & 0.4 & \\
\hline Damage exponent & $\kappa$ & 1.2 & \\
\hline \multicolumn{4}{|l|}{ Damage evolution parameters } \\
\hline Time step size (Iteration) & $\Delta t$ (day) & 1 & \multirow[t]{2}{*}{ Proposed } \\
\hline Cycles per day & $N$ (day) & 5000 & \\
\hline \multicolumn{4}{|l|}{ Stimulus parameters } \\
\hline Bone resorption rate & $\alpha_{R}$ & 0.035 & \multirow{6}{*}[14,19]{} \\
\hline Bone forming rate & $\alpha_{F}$ & 0.045 & \\
\hline Bone damage resorption rate & $\alpha_{D}$ & 0.035 & \\
\hline Stimulus reference value for bone resorption. & $S_{R}$ & 0.0025 & \\
\hline Stimulus reference value for bone formation. & $S_{F}$ & 0.007 & \\
\hline $\begin{array}{l}\text { Stimulus reference value for bone damage } \\
\text { resorption. }\end{array}$ & $S_{D}$ & 0.01 & \\
\hline Reference value of the transmitted signal. & $f_{i}^{R}$ & 0.1 & Proposed \\
\hline
\end{tabular}

Table 1. Material properties for bone used in the remodeling simulation 


\section{Results}

Figure 9a shows that in the case of the (NROC) model, the resorbed bone region corresponds to the completely damaged area located in the thin crack path. A significant difference can be observed between ROC and NROC models. Application of the proposed ROC model predicted a larger resorbed area corresponding to both the damaged area due to local excessive damage and its border that represents the region of the disrupted signal (Figure 9a). The NROC model predicted smaller resorbed region compared to ROC one. This can explained by the fact that the NROC model focus the resorption of the damaged sites only generating a small resorption of cavity.

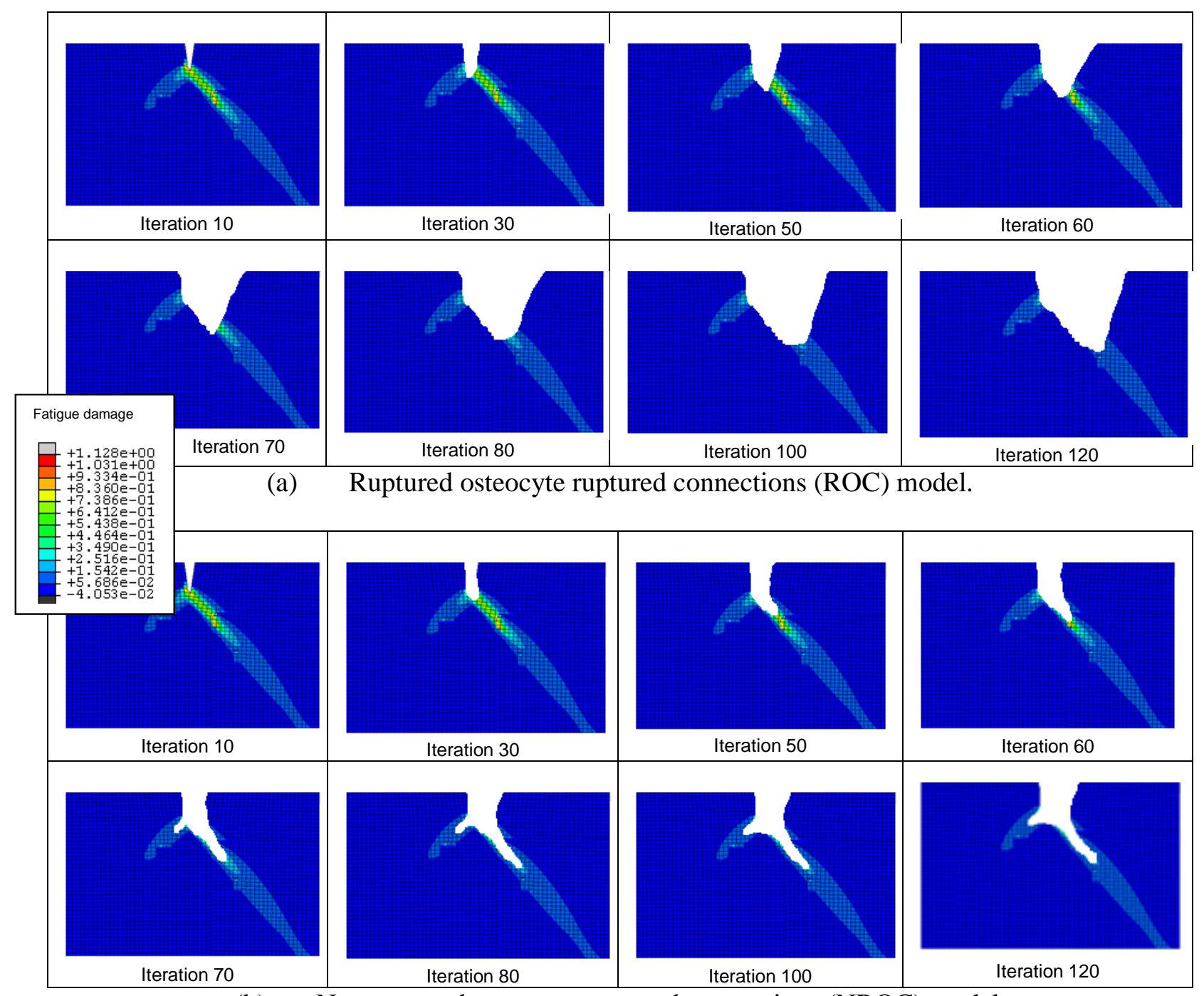

(b) Non ruptured osteocyte ruptured connections (NROC) model.

Figure 9. Predicted resorbed profiles at different iterations for two mechano regulation models (ROC and NROC). The contour represents the damage distribution. 
The current model demonstrated that introducing a fatigue microcrack alters the forming signals in bone and triggers resorption in the cracked area, suggesting that disruption of canalicular connections between osteocytes could provide the stimulus to initiate remodeling to repair damage in bone. In addition, it can be noticed that incorporating the surface condition resorption algorithm was necessary to simulate the creation of a resorption cavity at the surface of a bone to repair the damaged tissue located in the bone volume.

\section{Discussions}

Bone can repair itself. Small cracks, which grow under cyclic stresses by the mechanism of fatigue, can be detected and removed before they become long enough to be dangerous. Although assumed for a long time, the relationship between the locations of bone formation and resorption and the local mechanical environment is still under debate [30]. The lack of suitable imaging modalities for measuring bone formation and resorption in vivo has made it difficult to assess the bone formation and resorption experimentally. The experimental work of Burr et al. [31, 32] has shown that resorption cavities occur mainly in bone regions of microdamage in cortical and it is generally assumed that the same is true in cancellous bone. This occurs in the form of small cavities at microscale to remove the cracked bone. At this hierarchical level, microstructure of bone is formed by an arrangement of microfibriles, fibrils and fibers to form trabecular and cortical bone [33]. The microscopic fracture of bone tissue involves deformation and failure at multiple bone structural levels, ranging from the nanoscale mineral platelet/tropocollagen molecule interface, through microfibrils-fibrils-fiber scale, to crack propagation at microscopic scale (trabeculae and osteons). Such structural organization, generate material and structural anisotropy which may play a major role on (i) the fluid flow, (ii) the local fatigue microcracks initiation and propogation (iii) and the distribution of the osteocytes which sense the stimulus. All these factors may play a main role on the damage resorption due microcracks accumulation. 
Osteocytes are sensory cells which are capable of detecting the local mechanical and biochemical environment and conveying signals to each other and to other bone cells, thereby influencing the bone remodeling response. Knothe Tate et al. [34] proposed that a reduced osteocyte density in microdamaged bone may diminish the percentage of cells responsive to the mechanical loading of bone, thereby reducing the signaling to other bone cells on the surface. Also, the increased number of bone remodeling sites with overuse is preceded by programmed cell death in osteocytes $[9,10]$. The factors that initiate osteocyte apoptosis are not well understood, but may include direct damage to osteocytes via microcracks in the bone matrix or lack of convective fluid flow during disuse or disruption of the osteocyte processes. These findings support the idea that bone resorption due to damage accumulation may be related to the inhibitory signal transmitted through the osteocyte network. The proposed ROC model is an attempt to describe the inhibitory concept of damage.

The direct comparison between current predicted results with in vivo or ex vitro experimental data is very hard to achieve due to the difficulties to perform experiments related to the $3 \mathrm{D}$ network of osteocytes. Previous osteocytes network models $[17,18]$ were not able also to perform such a validation for the same reason.

The predicted results using the ROC model lead to a resorbed area that is larger than that of the conventional (NROC) model. Both the cracked zone with excessive damage and the region on its border representing the osteocyte network connections are removed. The obtained results based on the proposed damage-dependent distance function are in conformity with the conclusions presented in [35-37] suggesting that the mechanisms of bone formation and bone resorption are triggered at the bone cellular network level. An experimental study presented in [34] showed that osteocyte apoptosis is induced by bone fatigue and localized to the regions containing bone microcracks. Hazenberg et al. [2] have shown that microcracks in bone cause damage to the cellular material, by rupturing cell processes due to crack opening and shear displacements. The authors proposed that the number of broken cell processes may be a mechanism by which bone is able to estimate the crack size and orchestrate its response either by remodelling or by bone deposition on the outer surface. 
Hambli $[38,39]$ showed that differences between damage accumulation in tension and compression control the bone response. In the proposed model, a crack closure/opening damage parameter has been incorporated in the damage-dependent spatial-function to describe the effects of opening and closure of cracks within the bone.

The two widely used measures for the mechanical stimulus of bone are the magnitude of the shear stress created by a fluid passing over the cell at the microscopic level, and a macroscopic scalar function at whole bone level expressed in terms of the relationship between damage-strain-stress components. The development of this first ROC model for damage accumulation effects in osteocyte signalling networks leads to more realistic predicted bone resorption results in conformity with previously published ones.

Although many details of the mechanosensory system of the bone remodeling processes are still unknown, we have shown that a relatively simple regulatory bone remodeling based on the inhibitory signal transmitted due to damage accumulation may in some apects predict results in conformity with previously published results. Indeed, repetitive loading of bones has been shown to induce fatigue microdamage in the form of microcracks and areas of diffuse damage [39-41].

The existence of microdamage within bone has been reported to induce localized osteocyte apoptosis surrounding individual microcracks and that osteoclastic activity was over represented at sites of microdamage [39, 43] which subsequently leads to targeted remodeling. Martin, 2007 [44] suggested that the osteoclasts are able to sense and steer toward microdamage sites. Burr et al. [45] showed that within 8 days of the introduction of fatigue microdamage in canine radii by in vivo flexural loading, osteonal Basic Multicellular Unit (BMU) resorption spaces are found in proximity to microcracks about seven times more frequently than would be consistent with random associations. Experiments performed in rats by [37,46] demonstrated that the time dependent migration of osteoclasts into regions of cortical bone containing experimentally induced microdamage have strengthened the argument for the targeting of damage in bone.

In the present work, a finite element model that simulates trabecular bone resorption based on the inhibitory signal transmitted through the osteocyte network has been developed

The model is based on the idea that the osteocyte signal reduction is not related to the reduction of the stimulus sensed locally by osteocytes due to local damage but to the difficulties for the signal in 
travelling along a disrupted area. The concept has been suggested previously by several authors, but no finite element models have been developed to describe bone remodeling based on this idea. Here, a remodelling model based on a stimulus function coupled to a damage-dependent spatial function influence is proposed to describe the bone damage inhibitory effects in the osteocyte transmission network, including opening and closure of cracks within the bone.

The aim here was not to simulate the bone resorption for a real trabeculae. The focus was to mathematically develop the concept of bone resorption based on ruptured osteocytes connections and its implementation into a FE code to check its validity by comparing the obtained results to previously developed resorption model which is considered as the gold standard.

From a numerical point of view, the proposed model considers that every finite element of the mesh contains an osteocyte density and that these osteocytes are connected in the form of a network. Depending on the fatigue damage level, the signal transmitted within the osteocytes network is disturbed preventing the mechanical signal transmission which trigger in turn the bone resorption to repair fatigue damage in bone.

\section{References}

1. Marotti G., Cane V., Palazzini S. and Palumbo C., 1990. Structure-function relationships in the osteocytes. Ital. J. Electro. Metab., 93-106.

2. Hazenberg J.G., Freeley M., Foran A., Lee T.C. and Taylor D., 2006. Microdamage: A cell transducing mechanism based on ruptured osteocyte processes, J. Biomechanics, vol. 39, 2096-2103.

3. McNamara L. M., Prendergast J. P., 2007. Bone remodeling algorithms incorporating both stain and microdamage stimuli, J. Biomechanics, Vol. 40, (6), 1381-1391.

4. Cowin S.C., Moss M.L., 2001. Mechanosensory mechanisms in bone. In: Bone mechanics handbook, 2nd edn, chap 29, CRC Press, Boca Raton, 29-1-29-17.

5. Vassilios I. Sikavitsas, Johnna S. Temeno, Antonios G. Mikos, 2001. Biomaterials and bone mechanotransduction, Biomaterials 22, 2581-2593

6. Martin R. B.,2000. Toward a Unifying Theory of Bone Remodeling, Bone Vol. 26, (1), 1-6.

7. Kelly D.J. and Prendergast P.J., 2005. Mechano-regulation of stem cell differentiation and tissue regeneration in osteochondral defects, J. Biomech, Vol. 38, (7), 1413-1422.

8. Frost H.M., 1985, Bone microdamage: Factors that impair its repair. In: H.K. Uhthoff, Editor, Current Concepts in Bone Fragility, Springer, Berlin, 123-148.

9. Martin R. B, Burr D. R and Sharkey N. A,1998. Skeletal Tissue Mechanics. Springer, New York 
10. Noble B, 2003. Bone microdamage and cell apoptosis. Eur Cell Mater 21(6):46-55.

11. Martin R. B, 2003. Fatigue microdamage as an essential element of bone mechanics and biology. Calcif. Tissue Int 73(2):101-107.

12. Taylor D, Hazenberg JG, Lee TC, 2003. The cellular transducer in damage-stimulated bone remodelling: a theoretical investigation using fracture mechanics. J Theo. Biol. 225 (1): 6575.

13. Hambli R., 2014, Connecting mechanics and bone cell activities in the bone remodeling process: an integrated finite element modeling, Front. Bioeng. Biotechnol., 8;2:6.

14. Hambli R., Lespessailles E. and Benhamou C.L., 2013, Integrated remodeling to-fracture finite element model of human proximal femur behaviour, J Mech Behav Biomed Mater, 17:89-106.

15. Garcia-Aznar J. M., Rueberg T. and Doblare M., 2005. A bone remodelling model coupling microdamage growth and repair by 3D BMU-activity, Biom. Model Mechanobio 4: 147-167.

16. Parfitt, A.M., 1984. The cellular basis of bone remodelling: the quantum concept re-examined in light of recent advances in the cell biology of bone. Calcified Tissue Internation 36, S37S45.

17. Ausk B.J., Gross T.S. and Srinivasan S., 2006, An agent based model for real-time signaling induced ino steocytic networks by mechanical stimuli, Journal of Biomechanics 39, 26382646.

18. Jahani M, Genever PG, Patton RJ, Ahwal F, Fagan MJ, 2012, The effect of osteocyte apoptosis on signalling in the osteocyte and bone lining cell network: a computer simulation. $\mathrm{J}$ Biomech., 5;45(16):2876-83.

19. Hambli R., Soulat D., Gasser A. and Benhamou C.L., 2009, Strain-damage coupled algorithm for cancellous bone mechano-regulation with spatial function influence, Computer Methods in Applied Mechanics and Engineering, Vol. 198, Issues 33-36, 1, 2673-2682.

20. Chaboche J.L., 1981. Continuum damage mechanics-a tool to describe phenomena before crack initiation, Nucl Eng Des 64, 233-247.

21. Hernandez CJ (2001) Simulation of bone remodeling during the development and treatment of osteoporosis. PhD thesis, Stanford University, Stanford, CA

22. Mullender M.G. and Huiskes R., 1995. Proposal for the regulatory mechanism of Wolff's law. J. Orthop. Res. 13(4), 503-512.

23. Mikic B, Carter DR (1995) Bone strain gage data and theoretical models of functional adaptation. J. Biomech 28(4):465-469.

24. Dooley Dooley C, Tisbo P, Lee TC, Taylor D., 2012, Rupture of osteocyte processes across microcracks: the effect of crack length and stress, Biomech Model Mechanobiol.,11(6):75966.

25. Picandet V. 2001, Influence d'un endommagement mécanique sur la permeabilité et sur la diffusivité hydrique des bétons. Thesis dissertation in French, Nantes University.

26. Cowin, S.C., Weinbaum, S., Zeng, Y., 1995. A Case for Bone Canaliculi as the Anatomical Site of Strain Generated Potentials. Journal of Biomechanics 28, 1281-1297.

27. You J, Yellowley CE, Donahue HJ, Zhang Y, Chen Q, Jacobs CR. Substrate deformation levels associated with routine physical activity are less stimulatory to bone cells relative to loading-induced oscillatory fluid flow. Journal of Biomechanical Engineering 2000; 122:387393.

28. Vashishth, D., Verborgt, O., Divine, G., Schaffler, M.B. and Fyhrie, D. P. 2000, Decline in osteocyte lacunar density in human cortical bone is associated with accumulation of microcracks with age, Bone, 26, 375-80. 
29. Mullender MG, van der Meer DD, Huiskes R, Lips P. 1996, Osteocyte density changes in aging and osteoporosis, Bone;18(2):109-13

30. Schulte F.A., Ruffoni D., Lambers F. M., Christen D., Webster D. J., Kuhn G. and Muller R., 2013; Local Mechanical Stimuli Regulate Bone Formation and Resorption in Mice at the Tissue Level, PLoS ONE 8(4): e62172. doi:10.1371/journal.pone.0062172.

31. Burr, D.B., 'Remodeling and the repair of fatigue damage' Calcified Tissue International 53 (Suppl 1), S75 \}S81, 1993.

32. Burr, D.B., Hooser, M., 'Alteration to the en bloc basic fuschin staining protocol for the demonstration of microdamage produced in vivo', Bone 17, 431-433, 1995.

33. Hambli R. and Barkaoui A., 2012, Physically based 3D finite element model of a single mineralized collagen microfibril, Journal of Theoretical Biology, 21;301:28-41.

34. Knothe Tate, M. L., Adamson, J. R., Tami, A. E. and Bauer, T.W. 2004, The osteocyte, Int J Biochem Cell Biol, 36, 1-8.

35. Marotti G., 2000. The osteocyte as a wiring transmission system, J Musculoskel Neuron Interact; 1(2):133-136.

36. Li Y. Mi $Æ$ Mitra Basu $Æ$ Susannah P. Fritton Stephen C. Cowin, 2005. Analysis of avian bone response to mechanical loading, Part Two: Development of a computational connected cellular network to study bone intercellular communication. Biomechan Model Mechanobiol 4: $132-146$.

37. Verborgt O, Gibson GJ, Schaffler MB., 2000. Loss of osteocyte integrity in association with microdamage and bone remodeling after fatigue in vivo. J Bone Miner Res. 15: 60-67.

38. Hambli R., Multiscale prediction of crack density and crack length accumulation in trabecular bone based on neural networks and finite element simulation, International Journal for Numerical Methods in Biomedical Engineering, 2011 Vol.27, Issue 4, 461-475.

39. Ridha Hambli and Samir Allaoui, 2013, A robust 3D Finite element simulation of human proximal femur progressive fracture under stance load with experimental validation, Ann Biomed Eng.; 41(12):2515-27

40. Burr DB, Martin RB, Schaffler MB, Radin EL. Bone remodeling in response to in vivo fatigue microdamage. J Biomech.1985; 18: 189-200.

41. Burr DB, Milgrom C, Boyd RD, Higgins WL, Robin G, Radin EL. 1990. Experimental stress fractures of the tibia. Biological and mechanical aetiology in rabbits. J Bone Joint Surg $\mathrm{Br}$ 72B:370 - 375 .

42. Schaffler MB, Choi K and Milgrom C. 1995. Aging and matrix microdamage accumulation in human compact bone. Bone 17:521 - 525 .

43. Mori S, Burr DB. Increased intracortical remodeling following fatigue damage. Bone 1993;14:103-9.

44. Martin R.B., 2007, Targeted bone remodeling involves BMU steering as well as activation, Bone $40: 1574-1580$

45. Burr DB, Forwood MK, Fyhrie DP, Martin RB, Schaffler MS, Turner CH. Bone microdamage and skeletal fragility in osteoporotic and stress fractures. J Bone Miner Res 1997;12:6-15.

46. Noble BS, Peet N, Stevens HY, Brabbs A, Mosley JR, Reilly GC, Reeve J, Skerry TM, Lanyon LE (2003) Mechanical loading: biphasic osteocyte survival and targeting of osteoclasts for bone destruction in rat cortical bone. Am J Physiol Cell Physiol 284: C934C943. 